\title{
Designing and Constructing a Controlled-Flow Apparatus to Study the Effect of Surface Flow Velocity on the Quality of Electropolishing of Niobium
}

\author{
Tina Wang \\ Chemical and Biological Engineering Department \\ Princeton University \\ Princeton, New Jersey 08544 USA \\ Michael J. Kelley \\ Applied Science, Thomas Jefferson National Accelerator Facility \\ Newport News, VA 23606 USA
}

\begin{abstract}
The focus of this project was to design and construct a model controlled-flow apparatus to study electropolishing of one $\mathrm{cm}^{2}$ niobium coupons at surface flow rates typical of cavity processing. A simulation of the apparatus was constructed using CFDesign, a flow and thermal simulation software, to ensure that the selected dimensions may be expected to provide steady-state, laminar flow across the surface of the niobium coupon. Based on these dimensions, a sample system and apparatus was produced to determine the correct reservoir elevation heights for the desired flow rates for fluid viscosities represented in the mixed acid electrolyte. From the CFDesign simulations, it was found that the flow channel supplied laminar flow rates when the center of the niobium coupon was located $40 \mathrm{~mm}$ downstream from the inlet stream. The corresponding system, based on the CFDesign simulations, showed that the reservoir elevation heights for flow rates of $0 \mathrm{~cm} / \mathrm{s}$ to $5 \mathrm{~cm} / \mathrm{s}$ ranged from 0 to $1.27 \mathrm{~cm}$. The correlation between pressure heads and flow rates has been analyzed and an equation for flow rate was determined using experimental results. The detailed dimensions regarding the flow channel and information regarding the respective pressure heads serve as resources to finding the optimal flow rate for electropolishing the niobium cavities. Although previous research has found a correlation between the quality of electropolishing and internal surface flow rates, research facilities, including Jefferson Lab, did not have the equipment to pursue further analysis. Each nine-cell niobium cavity costs over $\$ 50,000$, so it is cost prohibitive to use real cavities to conduct early stage research. The prototype built through this research work provides a cost effective alternative. It can be used to validate some of the theoretical results obtained through simulation. In addition, the device allows for easy variable measurements that are either difficult or impractical with an enclosed niobium cavity, as sensors can be embedded into the device in the construction stage. Moreover, the data collected through our experiment furthers superconducting radiofrequency (SRF) technology by allowing Jefferson Lab to design a more effective electropolishing process.
\end{abstract}

\section{INTRODUCTION}

Much of the work at Thomas Jefferson National Accelerator Facility (Jefferson Lab) is devoted to the advancement of superconducting radiofrequency (SRF) technology for particle accelerators. Central to superconducting radiofrequency (SRF) technology are the multi-celled niobium cavities, which accelerate electrons by harnessing electric fields. Celled electropolishing is used to maximize the smoothness of the cavities' interior surface. Previous research has shown that the quality of electropolishing depends on variables such as surface flow 


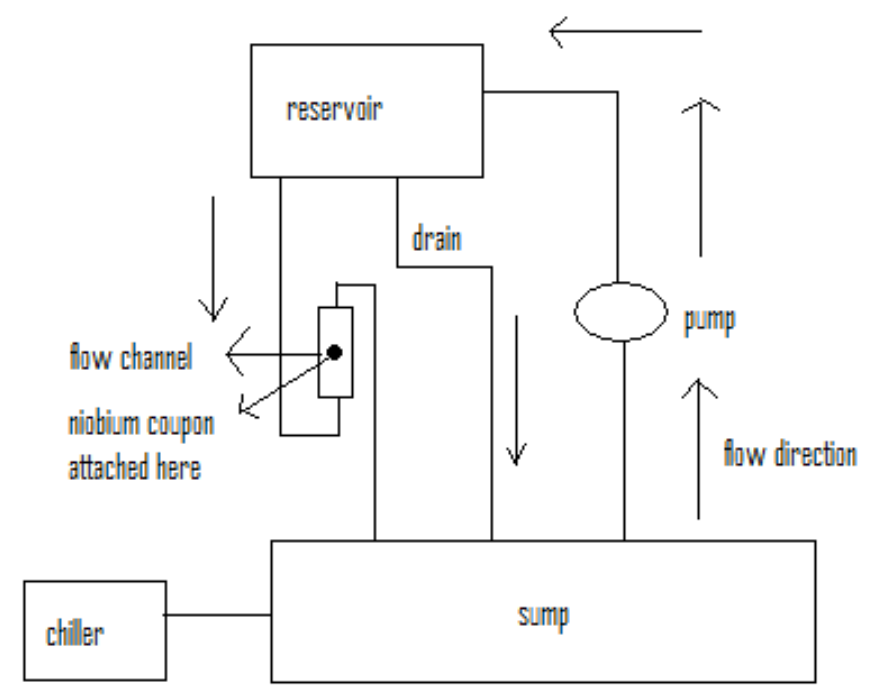

Figure 1. Simplified Flow Schematic.

rate and internal temperature. However, no one has tested the extent of the impact of varying flow rates on electropolishing.

The Continuous Electron Beam Accelerator Facility (CEBAF) superconducting radiofrequency accelerator is the principal research tool at the Jefferson Lab. The high energy beam from the accelerator fragments the nucleus of the target molecule, allowing scientists to look at the constituent quarks and gluons. The SRF microwave cavities drive the beam, and it is important to maximize their performance. The shallow radiofrequency penetration depth $(\sim 4 \mathrm{~nm})$ makes the cavity interior surface critical. Niobium has proven to be the most effective material for the cavities, due to its high critical temperature and critical electric field [1].

The smoothness of the cavity interior is critical to the efficiency of the accelerator. At Jefferson Lab, electropolishing is used to promote the smoothness of the niobium surface by eliminating surface peaks and defects. During electropolishing, an etchant-depleted mass transfer film covers the niobium surface. The local thickness of the film is dependent on the local topography of the niobium surface. The film is thickest at microdepressions (providing high electrical resistance) and thinnest at microprojections (providing low electrical resistance). Hence, protrusions are more quickly removed [2].
The film arises from the electropolishing mechanism, which leads to a reactant-depleted zone (film) in the electrolyte adjacent to the surface [1]. Hydrofluoric acid is typically the key reactant in the electropolishing process [3]. Electropolishing in the hydrofluoric acidsulfuric acid electrolyte may be summarized as [3]:

$$
2 \mathrm{Nb}+10 \mathrm{HF}+2 \mathrm{H}_{2} \mathrm{O} \rightarrow 2 \mathrm{H}_{2} \mathrm{NbOF}_{5}+5 \mathrm{H}_{2}
$$

Several studies have already been done to maximize the effectiveness of electropolishing. Currently, a mixture of hydrofluoric acid and sulfuric acid in a 1:9 volume ratio comprises the electrolyte [1]. However, scientists are interested in alternative solutions because hydrofluoric acid is known to be very hazardous to human health. Electropolishing can be done with a solution, in which methanol permits operation at high enough cell voltage for sulfuric acid to be used without HF [4]. The concentration of methanol is important. For instance, electropolishing at room temperature reaches its optimal effect with electrolytes at 0.1 molar concentration but is not adequately smooth. At a temperature of $-30{ }^{\circ} \mathrm{C}, 2$ molar concentration electrolytes produce the best electropolishing result [4]. The electropolishing reaction for the methanol-sulfuric acid mixture has not been discussed in the current literature. Based on 


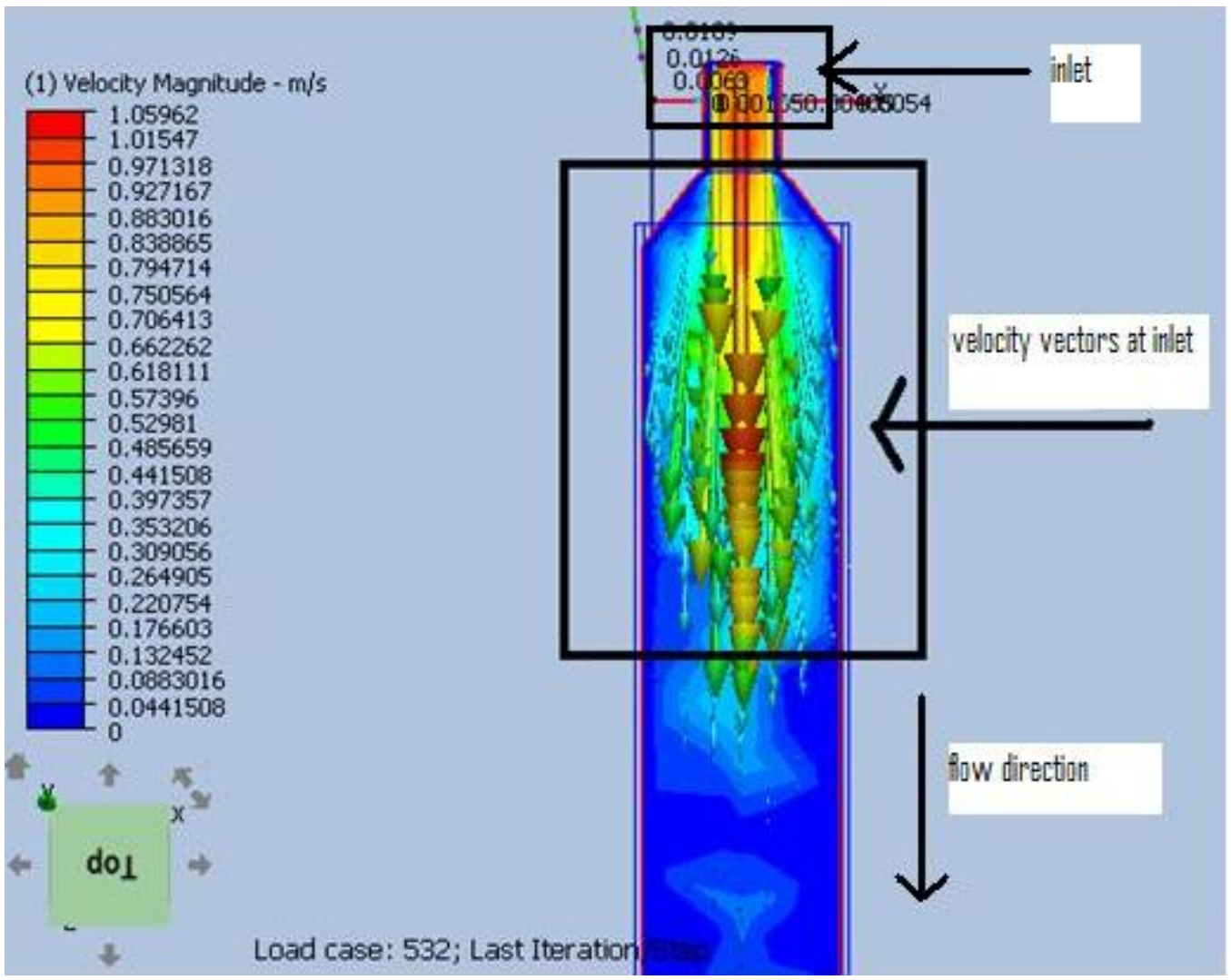

Figure 2. Velocity vectors at the inlet.

the hydrofluoric acid-sulfuric acid reaction, the reaction for the new mixture can be predicted as:

$$
\begin{aligned}
2 \mathrm{Nb}+10 \mathrm{CH}_{3} \mathrm{OH} & +2 \mathrm{H}_{2} \mathrm{O} \\
\rightarrow & 2 \mathrm{H}_{2} \mathrm{NbO}(\mathrm{OH})_{5}+5\left(\mathrm{CH}_{3}\right)_{2}
\end{aligned}
$$

Computational modeling of electropolishing indicates, and experiments have shown, that the temperature at the cavity walls is much higher than that in the electrolyte [5]. A full model of the thermal conditions throughout the cavities was produced using CFDesign [5]. The model showed that local temperatures and flow rates vary significantly inside the cavity. The study concluded that applying water cooling to the cavity exterior may help stabilize the thermal conditions. The scientists involved in this study noted that local flow also impacted the quality of electropolishing, and that future studies should consider its effect on surface topography.
The effect of surface flow rates during electropolishing has yet to be studied in detail as such studies require a controlledflow apparatus. The present study involves designing, building, and testing the first version of such an apparatus. The apparatus will support coupons of niobium and ensure constant, laminar surface flow at the coupon surface. Target surface flow rates range from $0 \mathrm{~cm} / \mathrm{s}$ to $5 \mathrm{~cm} / \mathrm{s}$, typical flow rates of electropolishing. Temperature will be controlled with a chiller attached to the sump. The model will test simulant fluids including water and motor oil. Based on the model, a fluoropolymer apparatus will be built. The fluoropolymer apparatus will test both the hydrofluoric and sulfuric acid mixture and the methanol and sulfuric acid mixture at varying flow rates. These experiments will determine the dependence of surface smoothness on the flow rates across the cavity surfaces during electropolishing. 


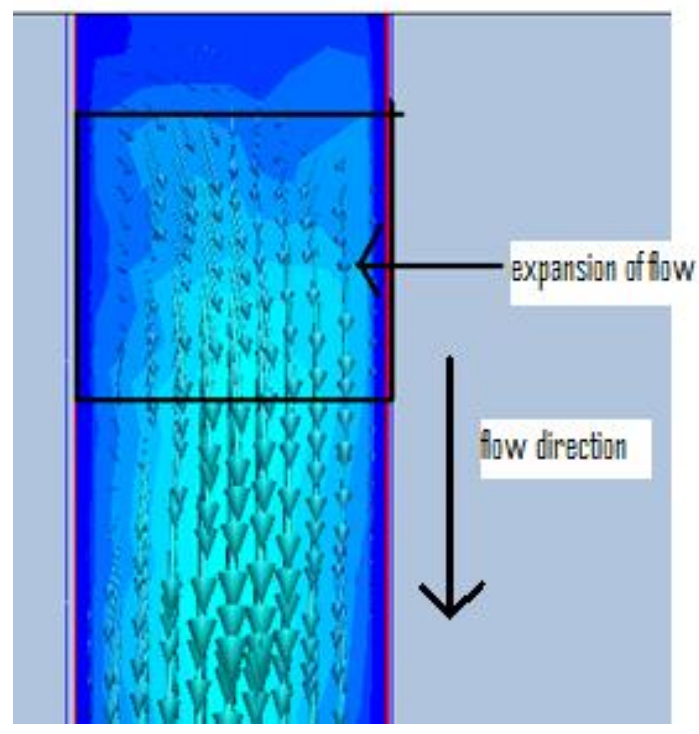

Figure 3. Velocity vectors near the entrance.

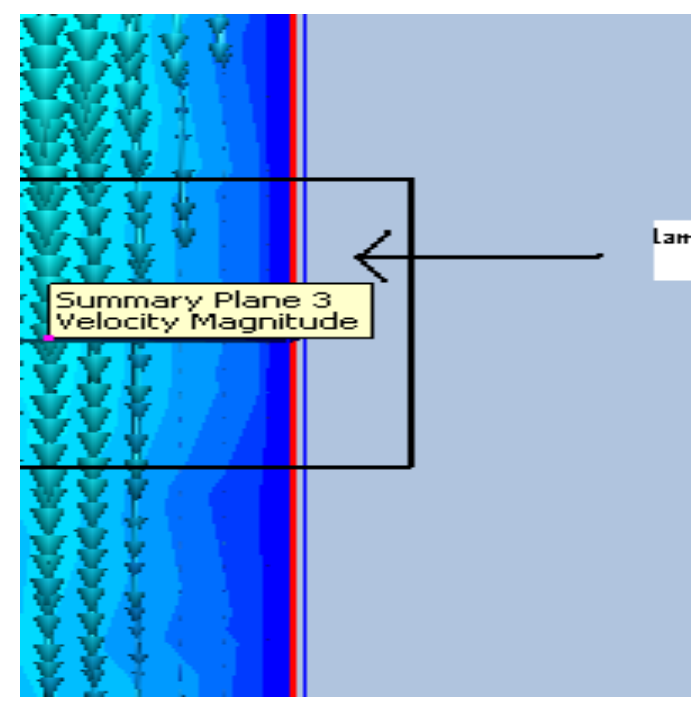

Figure 4. Velocity vectors approaching the specimen.

\section{METHODS}

\section{a. Process Concept}

The overall design of the flowcontrol system is shown in Figure 1. The flow channel is situated below a reservoir and above a sump. The electrolyte is initially poured into the sump. A pump moves the electrolyte from the sump to the reservoir. The reservoir is connected to the flow channel. The electrolyte moves from the flow channel to the sump. Additionally, the reservoir has an overflow back to the sump for the remainder of the electrolyte so as to maintain constant pressure head. A chiller is attached to the sump. The flow channel models vertical electropolishing but can be reoriented. The niobium coupon is attached to the side of the flow channel. One surface will be immersed in the electrolyte during electropolishing.

The dimensions of the flow channel were determined using computer simulation and modeling. The software CFDesign 2010 was used to produce simulated results. The apparatus needs to be long enough to accommodate the specimen, and, most importantly, ensure constant laminar flow across the specimen's surface. Due to the nature of piping, the apparatus is bound to see entrance effects in the flow. Initially, the flow would have to expand to the full crosssectional area, resulting in a diagonal flow pattern. If the specimen is too close to the entrance, the surface flow would not be at steady-state laminar. Such a design is undesirable, as flow patterns should be consistent in experiments to accurately assess the effect of flow rates on the quality of electropolishing. Hence, it is important that the specimen is placed at a location that experiences laminar flow rates even at the highest tested volumetric flow rates, which would be flow rates producing surface velocities of $5 \mathrm{~cm} / \mathrm{s}$.

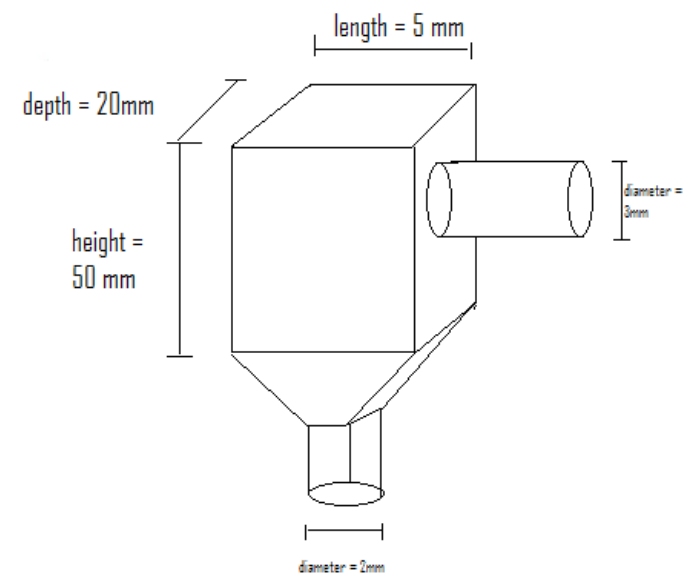

Figure 5. Design of Flow Apparatus. 


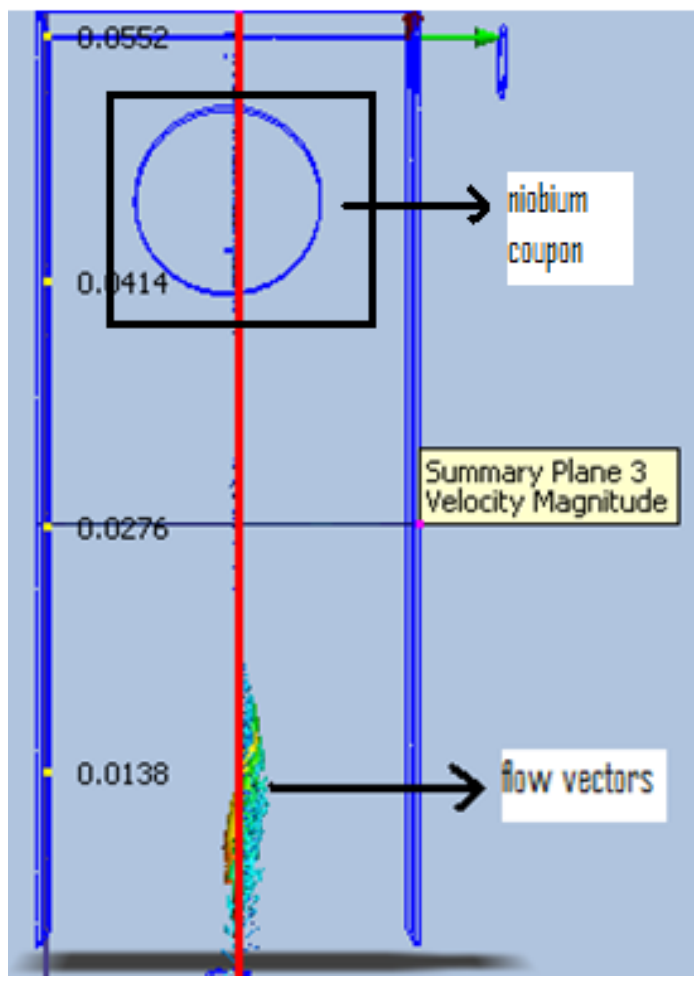

Figure 6. Velocity vectors of motor oil at entrance.

\section{b. Process Simulation}

Although designing a very long (infinitely long, for instance) apparatus would resolve the issue of entrance effects, such a design would restrict the choice of an effective pump. Hence, simulations are helpful at finding the proper balance in the trade-off between pump power and entrance effects. Because water is the safest liquid, it was chosen to be the first simulant fluid. In producing the simulations, various computer-aided design (CAD) files were produced. Each CAD file contained an apparatus with various dimensions. Materials and boundary conditions were assigned to each design. Proper volumetric flow rates were calculated according to the corresponding cross-sectional area. These flow rates were set to the boundary conditions. After all the conditions have been set, iterations were run. Five hundred iterations were set as the default; however, the iterations stopped once the results converge. Visual representations of the results were produced to analyze the flow paths of the water. Various planes were created to observe the flow rates in different parts of the apparatus. Velocity vectors helped visualize the direction of the water in the channel.

After studies using water as the simulant fluid were completed, additional simulant fluids were chosen. Due to the higher viscosity of the hydrofluoric acidsulfuric acid mixture (typically ranging from 20 to 40 centipoises), further simulations were completed using motor oil, having the viscosity of 50 to 100 centipoises (as opposed to the lower viscosity of water, at one centipoise), as the simulant fluid. The simulations were used to determine whether the flow channel dimensions would still provide laminar flows with the more viscous fluid. Because the methanol-sulfuric acid mixture is not yet commonly used, the current simulations and experiments focus on the hydrofluoric acid-sulfuric acid mixture.

\section{SIMULATION RESULTS AND DESIGN OF THE APPARATUS}

A selected set of simulation results are presented in order to demonstrate the design decisions for the design of the apparatus. First, Figures 2 and 3 show the velocity vector at the inlet and near the entrance. In Figure 2, the niobium coupon is located downstream of the range of the image. The flow is expected to become completely laminar at the location of the niobium coupon.

As can be seen in Figure 2, the flow initially expands outwards to fill up the body of the apparatus. Eventually, the expansion will be complete and the velocity vectors become vertical. Close observation of the velocity vectors at the surface of the specimen show the flow is constant, see Figure 3 . The verticality of the velocity vectors shows that flow is consistent and free of eddy currents throughout that region of the apparatus.

The CFDesign software shows that the flow across the specimen is laminar. In the simulation, a volumetric flow rate resulting in a velocity of $5 \mathrm{~cm} / \mathrm{s}$ is chosen. Theoretically, a laminar flow regime has a Reynolds number under 2300. Reynolds number is given in Equation 1:

$$
R e=\frac{\rho V^{2} L}{\mu}
$$




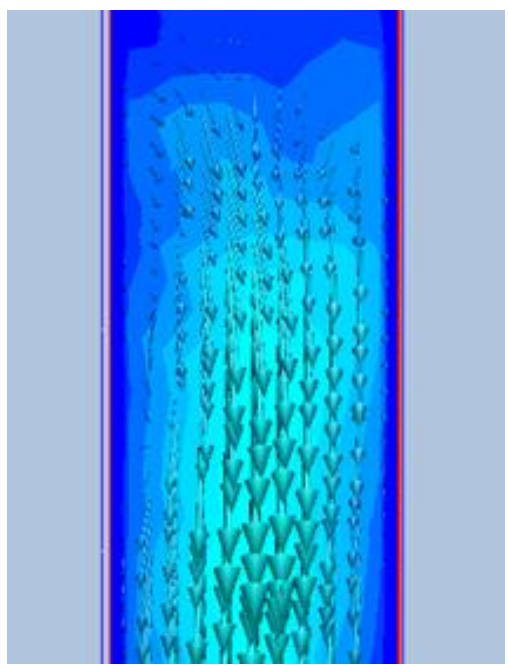

Figure 7. Velocity vectors of motor oil near the entrance.

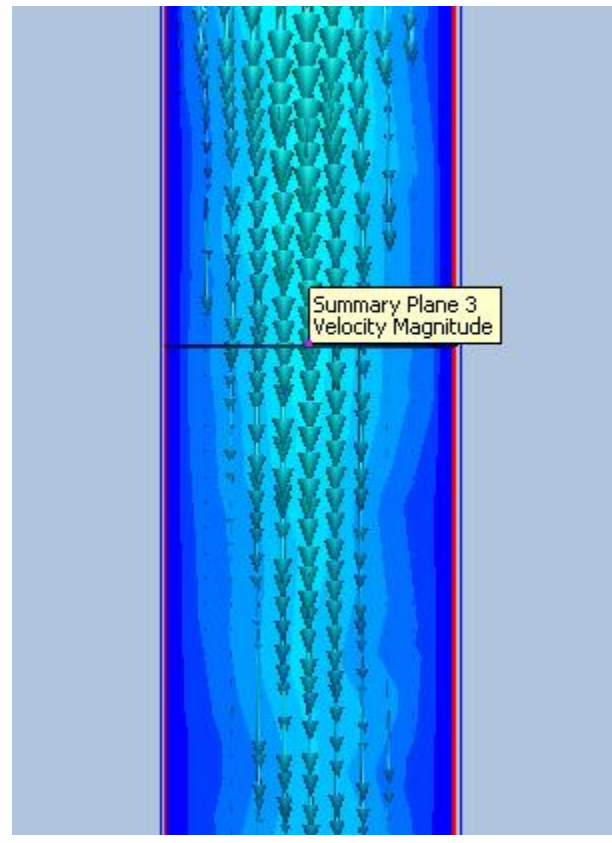

Figure 8. Velocity vectors of motor oil approaching the specimen.

Here, $V$ is the flow velocity of a single fluid particle, $L$ is the length of the channel, $\mu$ is the viscosity of the electrolyte, and $\rho$ is the density of the electrolyte. Since all the variables are fixed except for $V$, the velocities resulting in a laminar flow regime can be easily calculated. From Figure 4 , it is evident that the velocity vectors have reached laminar flow as they point straight down.
The simulations show that the water achieves steady-state laminar flow in the flow channel with the dimensions: height 50 $\mathrm{mm}$, width $5 \mathrm{~mm}$, and depth $20 \mathrm{~mm}$. The outlet pipe will have a diameter of $3 \mathrm{~mm}$ and the inlet pipe will have a diameter of $2 \mathrm{~mm}$, see Figure 5.

In further simulations using motor oil as the simulant fluid, the $20 \mathrm{~mm}$ by $50 \mathrm{~mm}$ by $5 \mathrm{~mm}$ model has proven to provide the desired steady-state, laminar flow. As seen in Figure 6, while the initial flow vectors show expansion patterns of motor oil as a fluid specimen, they reach steady-state far before reaching the specimen. In Figures 7 and 8 , the side view of the flow vectors shows the fluid expanding outwards (Figure 7) and then moving in a laminar fashion (Figure 8) before it moves out of the sight of the plane, thus indicating that the fluid is moving in a straight line alone the plane. The simulations using motor oil as the fluid confirmed that the determined dimensions (from the water simulations) for the flow channel were functional for the higher viscosity fluid.

From Equation 1, it can be derived that:

$$
\frac{\left(\frac{\rho}{\mu}\right)_{1}}{\left(\frac{\rho}{\mu}\right)_{2}}=V_{2}^{2} / V_{1}^{2}
$$

Equation 2 assumes the same characteristic length for the two apparatuses, which will be true since the same apparatus is used. Hence, a velocity for other fluids can be selected so that it has the same Reynolds number as water.

Based on the design shown in Figure 1, a physical model apparatus was constructed. A pipe with the same length and cross-sectional area as the flow channel in the simulation acted as the channel in the apparatus. The model apparatus will serve as the basis as the fluoropolymer apparatus to be built later at Jefferson Lab. The fluoropolymer apparatus will test the hydrofluoric acid-sulfuric acid electrolytes. Because the same apparatus is used for all experiments, the surface roughness of the boundary layer does not affect the results. Additionally, the thickness of the boundary layer does not impact the measurements because only the internal dimensions are important. 


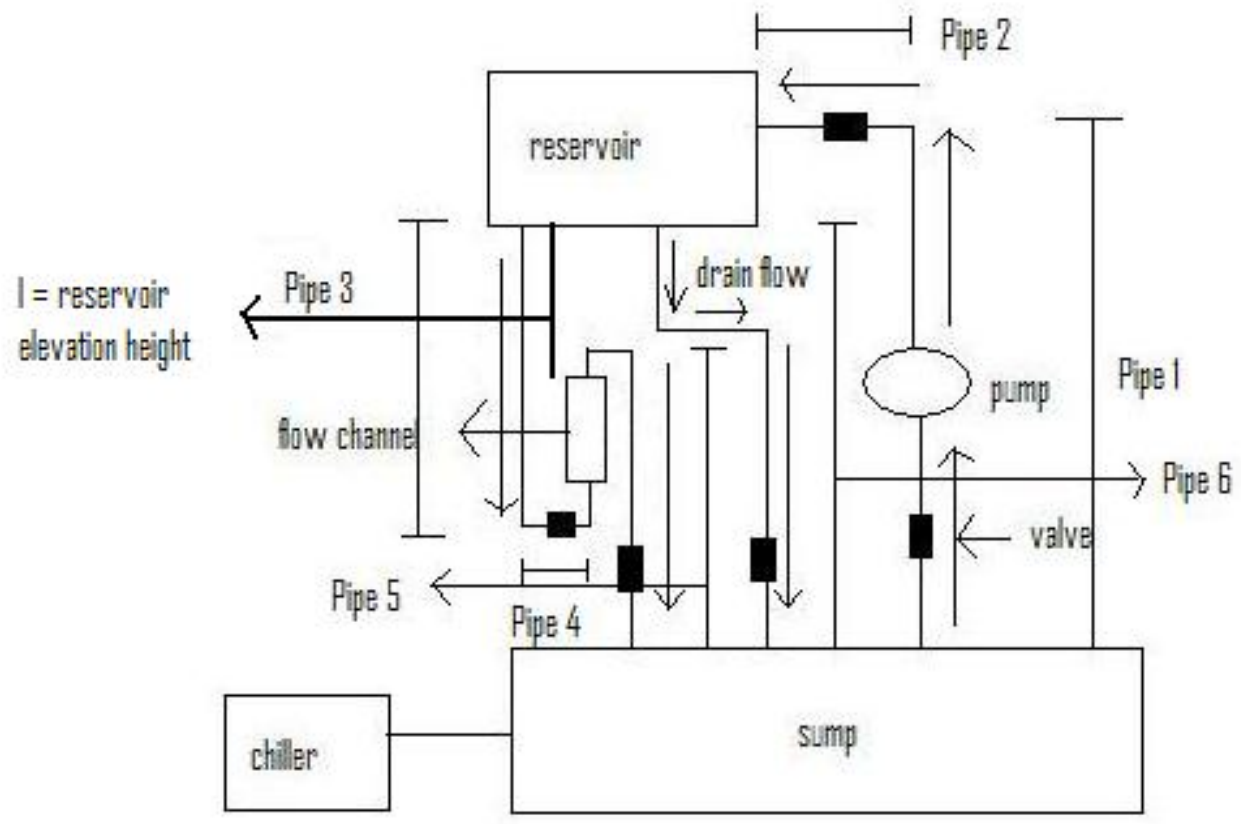

Figure 9. Design of Flow System; arrows show the direction of the electrolyte flow.

\section{EXPERIMENTAL DESIGN}

After a design was selected from the simulations and a preliminary physical model version of the apparatus and its system was built, water and antifreeze were used as the trial run simulations fluids. The two fluids were selected because they would test flow patterns at different fluid viscosities. Water has a viscosity of one centipoise at room temperature whereas antifreeze has a viscosity of fifteen centipoises: close to the viscosity of the hydrofluoric acid-sulfuric acid mixture. A pipe with the same length and cross-sectional area served as the flow channel in the test runs. The flow of simulant fluids through the apparatus is shown in
Figure 9 while Figure 10 shows the specific lengths of the pipes with corresponding diameters.

To obtain the desired surface flow rate across the specimen, the dimension $l$ (length), the reservoir elevation height in Figure 9, was varied. A container was placed within the sump to carry the liquid that escapes from the flow channel. The time for each flow session was taken. Volumetric flow rate can then be calculated based on the amount of fluid in the container divided by the time recorded. Volumetric flow rates at each $l$ were taken. The corresponding surface flow rate was calculated using the following equation:

\section{Surface flow rate $=$ Volumetric flow rate/cross-sectional area of the flow channel}

The relationship between the reservoir elevation height, which is directly related to the pressure head, is defined by Equation 4, derived from Bernoulli's equation:

$$
\frac{v^{2}}{2 g}=\varphi+z
$$

Here, $v$ is the electrolyte's flow velocity, $g$ is the acceleration due to gravity, $\varphi$ is the pressure head in terms of the difference between the column elevation and the piezometer bottom, and $z$ is the elevation at the piezometer bottom. In Figure 9, the $\varphi+z$ term should be 


\begin{tabular}{|c|l|l|}
\hline & Diameter & Length \\
\hline Pipe 1 & $0.953 \mathrm{~cm}$ & Variable \\
\hline Pipe 2 & $0.953 \mathrm{~cm}$ & Variable \\
\hline Pipe 3 & $0.535 \mathrm{~cm}$ & Variable \\
\hline Pipe 4 & $0.451 \mathrm{~cm}$ & $10.15 \mathrm{~cm}$ \\
\hline Pipe 5 & $0.431 \mathrm{~cm}$ & $27.46 \mathrm{~cm}$ \\
\hline Pipe 6 & $0.935 \mathrm{~cm}$ & Variable \\
\hline
\end{tabular}

Figure 10. Design of Flow System with Pipe Measurements.

equivalent to the reservoir elevation height, or $l$.

According to Equation 3, it is expected that the fluid velocity would be proportional to the reservoir elevation height. As the elevation of the reservoir decreases, less pressure head is available, hence reducing the flow velocity.

\section{MEASUREMENT AND DATA ANALYSES}

Using the measurements from the CFDesign simulations, the computed dimensions for surface flow velocities ranging from $0 \mathrm{~cm} / \mathrm{s}$ to $5 \mathrm{~cm} / \mathrm{s}$ can be found. The data presents a general correlation that relates $l$ (reservoir elevation height) to the desired flow rate.

Ten trials were taken for various reservoir elevation heights. The surface flow rate showed a decreasing trend with decreasing elevation heights. The median, mean, and standard deviations of the flow rate at each elevation were calculated. Graphs depicting the dependence of flow rates $(y)$ on elevation height $(x)$ are shown in Figures 11 and 12.

The linear trendlines serve as approximation tools for obtaining desired flow velocities. The median is generally more accurate because it does not become skewed due to extreme outliers. Therefore, the flow rate should generally be predicted as:

$$
v=0.3172 * l+5.9137
$$

Where $v$ is the flow rate in $\mathrm{cm} / \mathrm{s}$ and $l$ is reservoir elevation height in $\mathrm{cm}$. shown that:

Based on Equation 4, it can be

$$
v^{2}=2 g(\varphi+z)
$$

Here, it is shown that the squared value of the fluid velocity should be a linear function of the reservoir elevation height. The corresponding trendlines had higher $R^{2}$ values, indicating that this may be a more accurate prediction. The trends are not perfectly linear due to experimental errors to be discussed later. The graphs depicting the relationship between the squared values of velocity and reservoir elevation height are shown in Figure 13.

Using the median correlation, the flow rate can be predicted with:

$$
v=(2.2484 * l+26.363)^{1 / 2}
$$

a. Results

Trials were conducted with antifreeze as the simulant fluid. With the higher viscosity fluid, the flow rate at each reservoir elevation height was reduced by the mean ratio of one to 7.30 (water flow velocity to antifreeze flow velocity). Antifreeze has a viscosity of 15 centipoises, close to the lower bound of the electrolyte's viscosity at 20 centipoises. The median flow rates at reservoir elevation heights ten inches, nine inches, and eight inches were $2.095 \mathrm{~cm} / \mathrm{s}, 1.679 \mathrm{~cm} / \mathrm{s}$, and $1.734 \mathrm{~cm} / \mathrm{s}$, respectively. The linear 


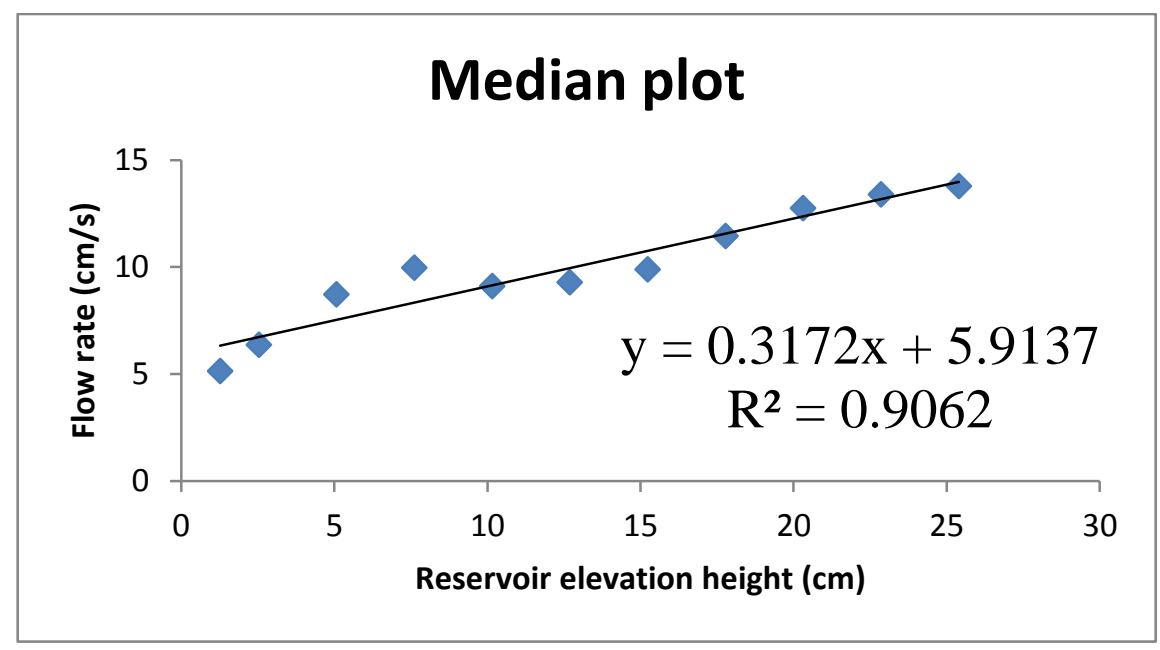

Figure 11. Median values of flow rates at various elevation heights.

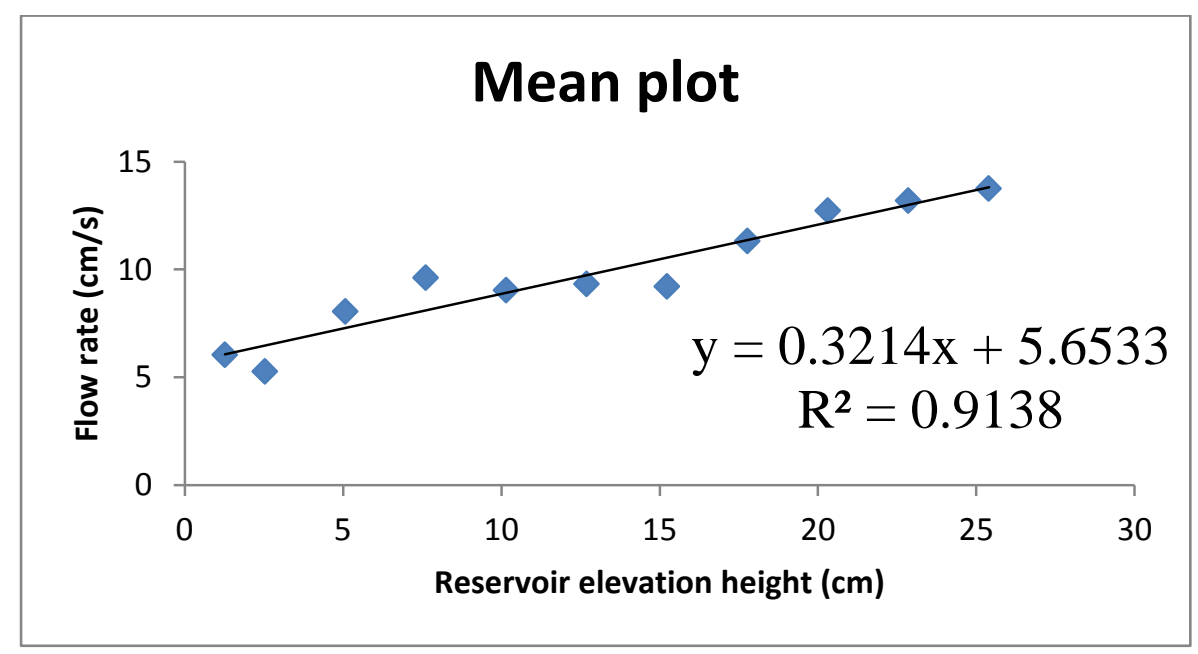

Figure 12. Mean values of flow rates at various elevation heights.

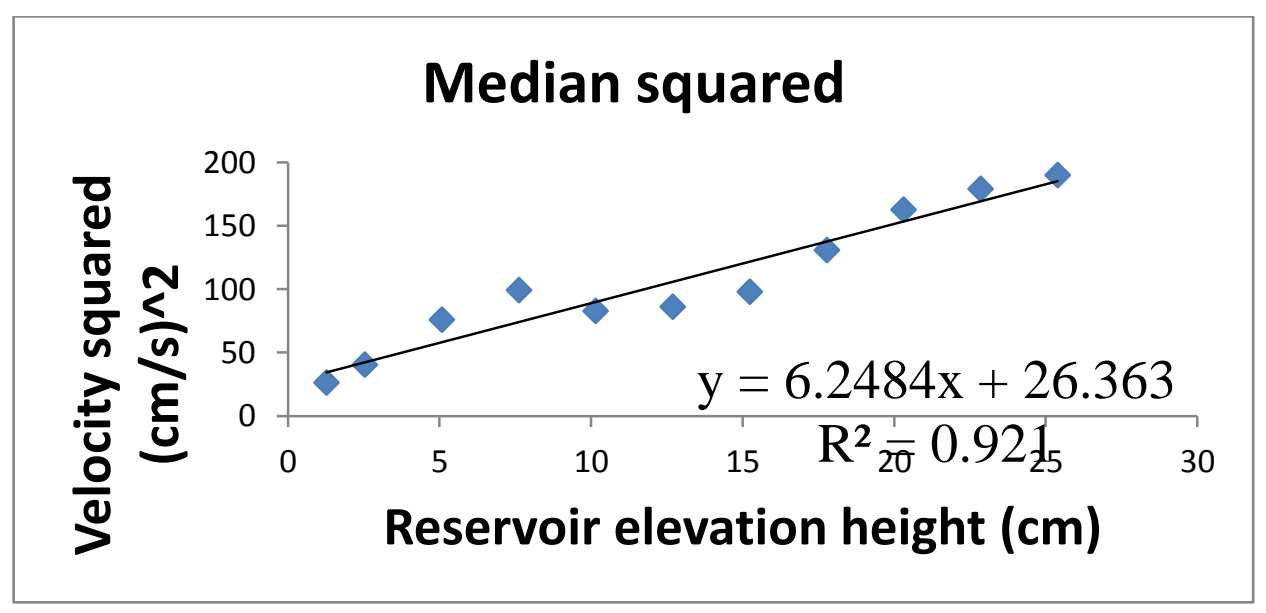

Figure 13. Median velocity squared vs. reservoir elevation height. 


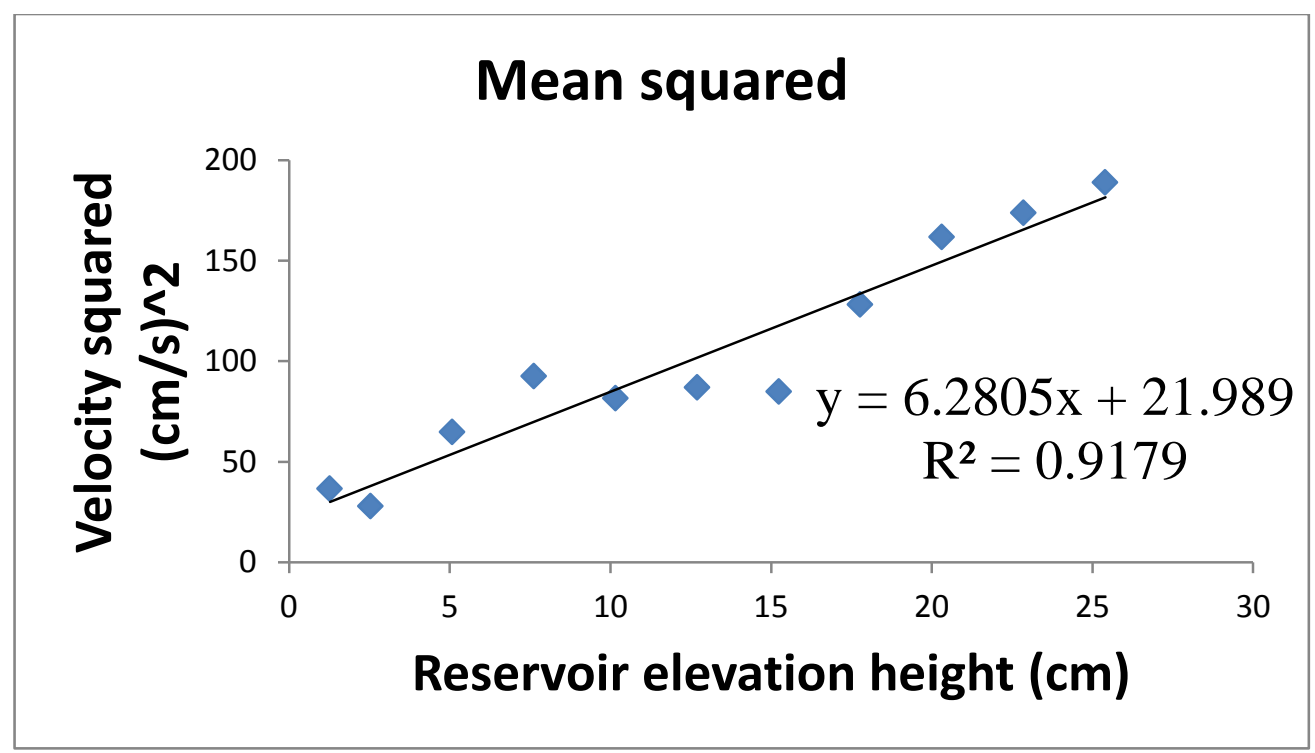

Figure 14. Mean velocity squared vs. reservoir elevation height.

trendline for prediction flow rates of antifreeze (or any fluid with viscosity of 15 centipoises) is:

$$
v=0.0711 * l+0.2103
$$

\section{b. Error sources}

Several conditions served as sources of error in the experiment. The changes in the diameter of the inlet stream resulted in occasional air bubbles forming in the flow channel. When an air bubble was present, the pipes needed to be bent and readjusted before the apparatus could function properly. In these cases, the times recorded were generally shorter than the actual time due to the delay from rearranging the apparatus. Additionally, the beaker used to measure the volume of water for a given trial was not exact due to human error in reading measurements. Lastly, human reaction time affected the time recorded.

\section{CONCLUSIONS}

Simulations were used to determine the optimal dimensions for the flow apparatus. A preliminary flow-control apparatus and a final model of the flow channel were built. Based on the CFDesign simulations, a model apparatus was constructed and measured flow were made. A flow-control apparatus can be built out of fluoropolymer from this data. The flow velocity can be changed by adjusting the elevation height of the reservoir. Alternatively, the flow velocity can be changed via changing the liquid level in the reservoir. This manipulation can be achieved with a valve downstream of the reservoir and at the discharge of the pump, both of which have already been incorporated in the flow schematic. The computational model, created using CFDesign, and the sample system provide an effective design for a flow-control apparatus. The fluoropolymer apparatus will be based on the constructed model and will test hydrofluoric acid-sulfuric acid and methanol-sulfuric acid solutions. The apparatus will determine the optimal flow rate for electropolishing. Operating the electropolishing process at the optimal flow rate would help obtain far more effective active layers in the niobium cavities, allowing the superconducting radiofrequency accelerator to operate more efficiently. Because the fluoropolymer apparatus model would cost over $\$ 10,000$ to build, it is critical that a preliminary apparatus is designed, built, and tested first. The overall saving achieved by this work is more significant as using an actual nine-cell cavity would cost over $\$ 50,000$. 


\section{ACKNOWLEDGMENTS}

The research work was funded by Department of Energy and conducted at Thomas Jefferson National Accelerator Facility (Jefferson Lab). Dr. Michael J. Kelley, researcher at Jefferson Lab and professor at the College of William and Mary, served as the mentor of the project. The author would like to thank the members of Jefferson Lab for their support during the entire process.

\section{REFERENCES}

1. H. Tian, S. G. Corcoran, C. E. Reece, and M. J. Kelley, "The Mechanism of Electropolishing of Niobium in Hydrofluoric-Sulfuric Acid Electrolyte," Journal of the Electrochemical Society, Vol. 155 (9), pp. 563-568, July 2008.

2. Electropolishing: A User's Guide to Applications, Quality Standards, and Specifications. $9^{\text {th }}$ Ed. Jan. 2003. Houston, Texas. Delstar Metal Finishing, Inc.

3. J. R. Delayen, J. Mammosser, L. Phillips, and A. T. Wu, "Alternative Electrolyte Composition for Electropolishing of Niobium Surfaces," Jefferson Lab publication 2001.

4. X. Zhao, S. G. Corcoran, M. J. Kelley, "Electropolishing of Niobium in Sulfuric Acid-Methanol Electrolytes for Radiofrequency Superconducting Accelerator Cavities Fabrication," J. Electrochemistry Society.

5. C. E. Reece, J. Ortega, and J. Mammosser, "Hydrodynamic Thermal Modeling of 9-Cell ILC Cavity Electropolishing and Implications for Improving the EP Process," Prof. $13^{\text {th }}$ Superconducting Radiofrequency Workshop, pp. 275-279, Beijing 2007.

6. J. Hogan, T. Cannella, E. F. Daly, M. Drury, J. Fischer, T. Hiatt, P. Kneisel, J. Mammosser, J. Preble, T. Whitlatch, K. Wilson, and M. Wiseman, "SRF Accelerator Technology Transfer Experience from the Achievement of the SNS Cryomodule Production Run," Particle Accelerator Conference, Knoxville, Tennessee 2005.

7. J. R. Delayen, L. R. Doolittler, C. E. Reece, "Operational Optimization of Large-Scale SRF Accelerators," Particle
Accelerator Conference proceedings, New York 1999.

8. H. Tian, S. G. Corcoran, M. J. Kelley, C. E. Reece, "Novel Characterization of the Electropolishing of Niobium with Sulfuric Acid and Hydrofluoric Acid Mixtures," Superconducting Radiofrequency proceedings, pp. 370-376, 2007.

9. CFDesign 2010, Blue Ridge Numerics, Inc.

\section{Thomas Jefferson National Accelerator} Facility (Jefferson Lab)-an aerial view:

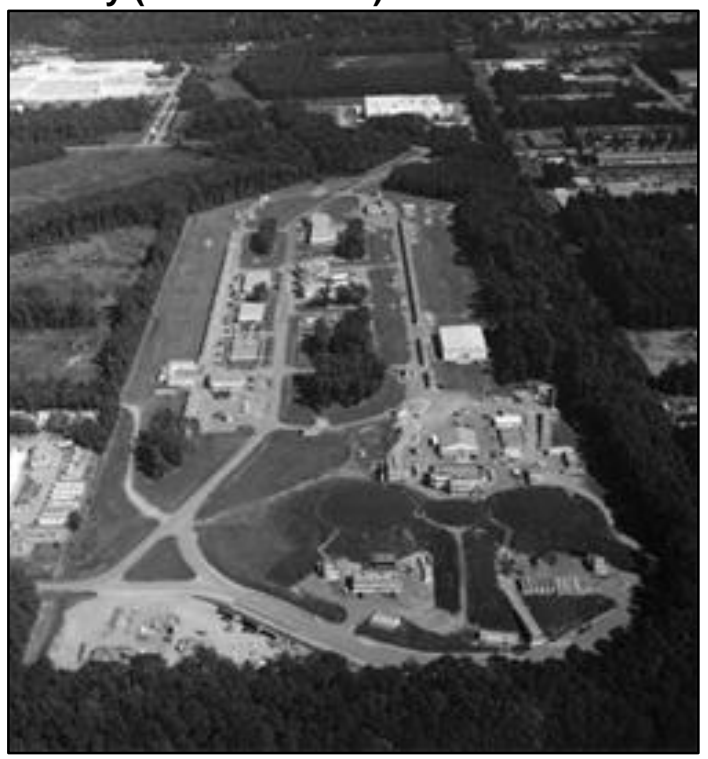

The Thomas Jefferson National Accelerator Facility (Jefferson Lab) is funded by the U.S. Department of Energy's Office of Science with strong support from the City of Newport News and the Commonwealth of Virginia. As a user facility for scientists worldwide, its primary mission is to conduct basic research of the atom's nucleus at the quark level.

With industry and university partners, Jefferson Lab also has a derivative mission: applied research for using the Free-Electron Lasers based on technology developed at the lab to conduct physics experiments. Additionally, as a center for both basic and applied research, Jefferson Lab reaches out to help educate the next generation in science and technology.

Jefferson Lab is managed and operated for the DOE by the Jefferson Science Associates, LLC. JSA is a limited liability corporation created by Southeastern Universities Research Association and Computer Sciences Corp. specifically to manage and operate Jefferson Lab. 


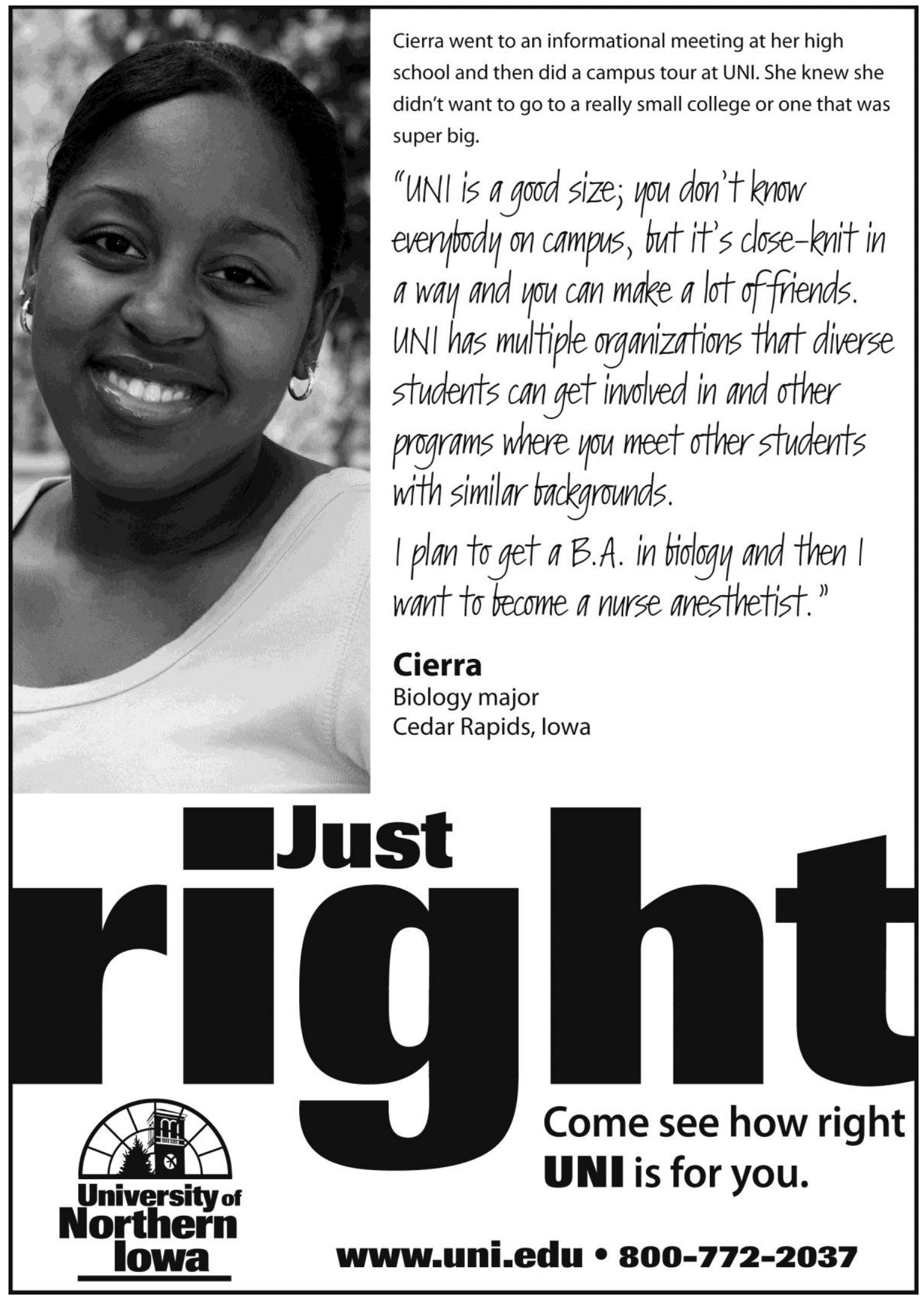

